Casos Clínicos

Arch. Esp. Urol. 2009; 62 (9): 755-757

\title{
HERNIA URETERAL INGUINAL: CASO CLÍNICO
}

Antón Zarraonandia Andraca, Ángel Ríos Reboledo?' Javier Casas Nebra, José Ponce Díaz-Reixa, Sara Martínez Breijo, Juan Gonzalez Dacal, Javier Sánchez R. Losada y Venancio Chantada Abal.

Servicio de Urología y Servicio de Radiología'. Complejo Hospitalario Universitario A Coruña. La Coruña. España.

Resumen.- OBJETIVOS: Describir caso clínico de hernia ureteral inguinal y luego comentar brevemente tema.

MÉTODOS: Paciente 80 años hipertenso con hernia inguinal izquierda operada, hemicolectomia derecha por adenocarcinoma de colon y colecistectomia. En urografía intravenosa como hallazgo se identifica uréter derecho en posición ectópica que sale de la cavidad abdominal por región inguinal derecha para luego volver al abdomen.

RESULTADOS: Se realiza TAC que confirma diagnostico. Debido a la edad avanzada del paciente, comorbilidades y ausencia de síntomas, se decidió actitud expectante.

CORRESPONDENCIA

Antón Zarraonandia Andraca Servicio de Urología Complejo Hospitalario Universitario A Coruña.

Xubias de Arriba, 84 15006 La Coruña. (España) antonzarraonandia@yahoo.com.

Trabajo recibido: 2 de mayo 2009. 
CONCLUSIONES: Las hernias urterales a través del conducto inguinal son poco frecuentes. Existen 2 tipos: paraperitoneal y extraperitoneal. La más frecuente es la paraperitoneal (80\%) donde se acompaña de saco herniario y pueden aparecer otros órganos intra abdominales. La extraperitoneal suele acompañarse de grasa retroperitoneal. Ambos tipos de hernias suelen se indirectas. El tratamiento suele ser la herniorrafia.

Palabras clave: Ureter. Hernia. Inguinal.

Summary.- OBJECTIVES: To describe a clinical case of ureteral inguinal hernia and to comment briefly about the topic.

METHODS: 80 year-old patient with, hypertension, left inguinal hernia sugery, right hemicolectomy for colon adenocarcinoma and cholecystectomy. Intravenous urogram casually found that the right ureter was leaving the abdominal cavity though the right inguinal duct and then returned to the abdomen.

RESULTS: CT scan confirmed the diagnosis. Due to the advanced age of the patient, comorbidities and the absence of symptoms, conservative treatment was decided.

CONCLUSIONS: Ureteral hernias through the inguinal duct are uncommon. There are 2 types: paraperitoneal and extraperitoneal. The most common is the paraperitoneal (80\%) which is accompanied by hernia sac and can appear with other abdominal organs. The extraperitoneal often accompanied by retroperitoneal fat. Both types of hernias are usually indirect. Treatment is usually herniorrhaphy.

Keywords: Ureter. Inguinal. Hernia.

\section{INTRODUCCIÓN}

Paciente varón de 80 años con antecedentes de hipertensión arterial y hernia inguinal izquierda operada, hemicolectomia derecha por adenocarcinoma de colon y colecistectomia.

Valorado en consultas de urología por dilatación leve del riñón derecho en ecografía abdominal de control, indicándose urografía intravenosa en agosto del 2008 donde se aprecia buena concentración de contraste, retraso de eliminación del contraste por sistema colector derecho que comienza a eliminar a los 45 minutos y dilatación del sistema pielocalicial derecho. Se identifica posición ectópica del uréter derecho que sale de la cavidad abdominal por región inguinal derecha para luego volver al abdomen (Figura 1).

Se realiza TAC de abdomen y pelvis con reconstrucción coronal donde se aprecia herniación de uréter dere- cho en canal inguinal (Figuras 2 y 3 ). Actualmente, el paciente esta asintomático desde el punto de vista urológico y presenta buena función renal (creatinina 1.1 $\mathrm{mg} / \mathrm{dL}$ ). Se decide tratamiento expectante con controles periódicos.

\section{DISCUSIÓN}

La herniación del uréter a través del conducto inguinal es poco habitual (1) alrededor de 140 casos reportados en la literatura (2). Esta patología puede afectar tanto a hombres como mujeres, siendo en los hombres más frecuente asociado con hernias inguinales y en mujeres con hernias femorales (3) y dado a que estas hernias suelen ser de gran tamaño, rara vez tienen complicaciones isquémicas. Otro sitio poco frecuente de herniación ureteral es el agujero ciático con 17 casos publicados en la literatura (4). Suelen aparecer síntomas urológicos cuando se asocia con herniación vesical, que pueden ser: aumento de frecuencia miccional, nicturia, disuria $y$ hematuria.

Es frecuente encontrar alteraciones en los estudios por imagen destacando uropatía obstructiva de grado variable. La clasificación de este tipo de hernia va a depender de la presencia o no de saco herniario. La más

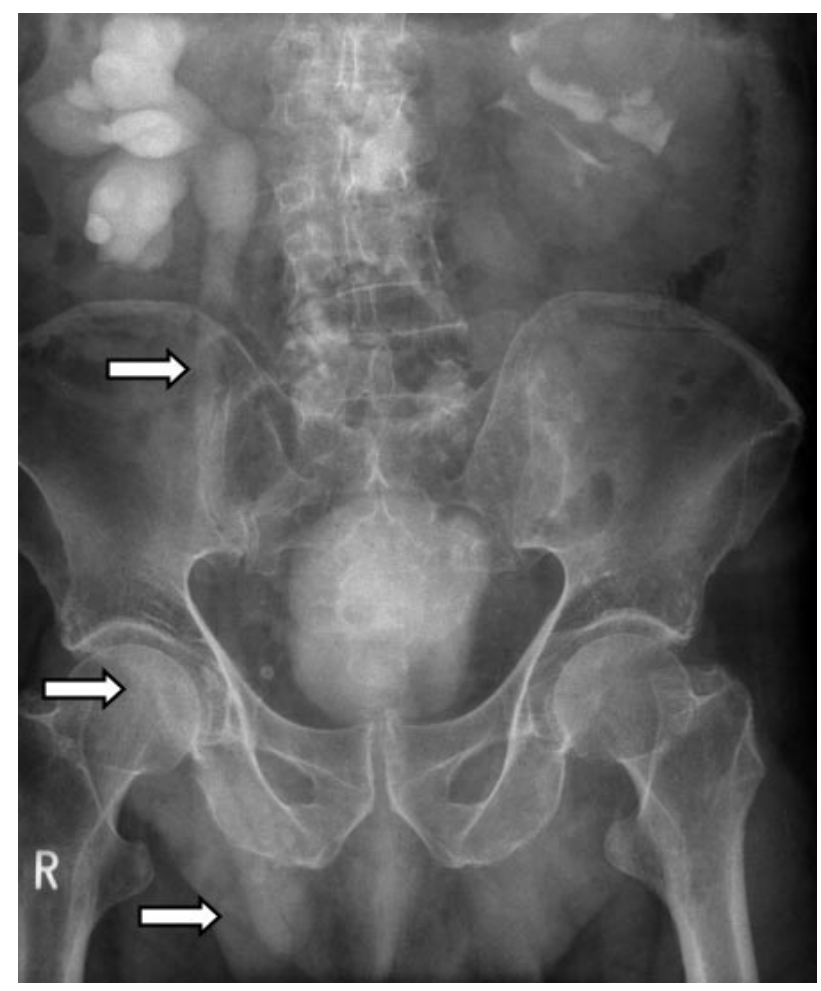

FIGURA 1. Urografía intravenosa 45 minutos post administración de contraste. 


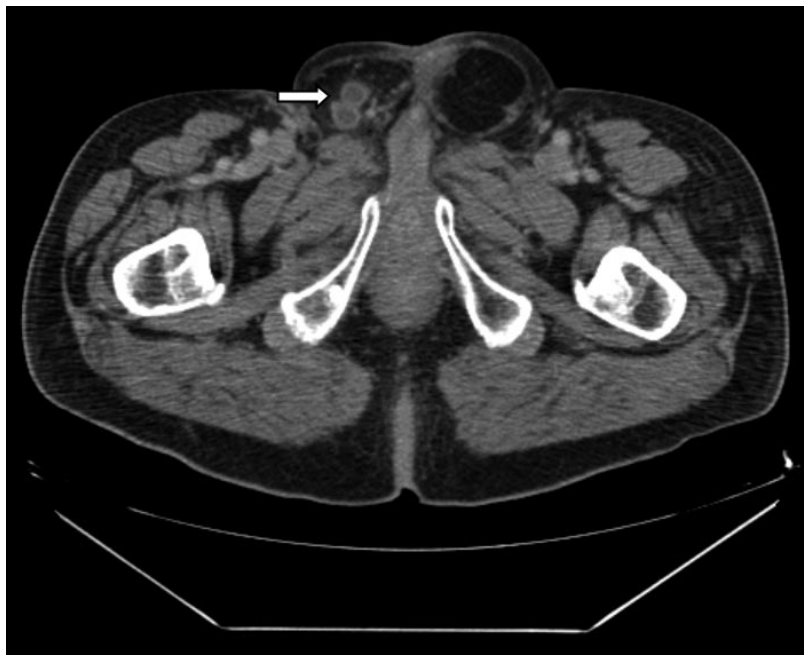

FIGURA 2. Corte axial TAC.

frecuente es la paraperitoneal $(80 \%)$ donde se acompaña de saco herniario y pueden aparecer otros órganos intra abdominales. La extraperitoneal suele acompañarse de grasa retroperitoneal (5). Ambos tipos de hernias suelen se indirectas (6).

La mayoría de las veces el diagnóstico es un hallazgo durante la cirugía o urografía (7). La herniorrafía está indicada para resolver la uropatía obstructiva, y es necesario hacer una disección precisa del uréter para liberarlo de estructuras vecinas, luego una reducción simple bastaría para resolver el problema (3).

En nuestro caso, dada la edad avanzada del paciente, comorbilidades y ausencia de síntomas, se decidió actitud expectante.

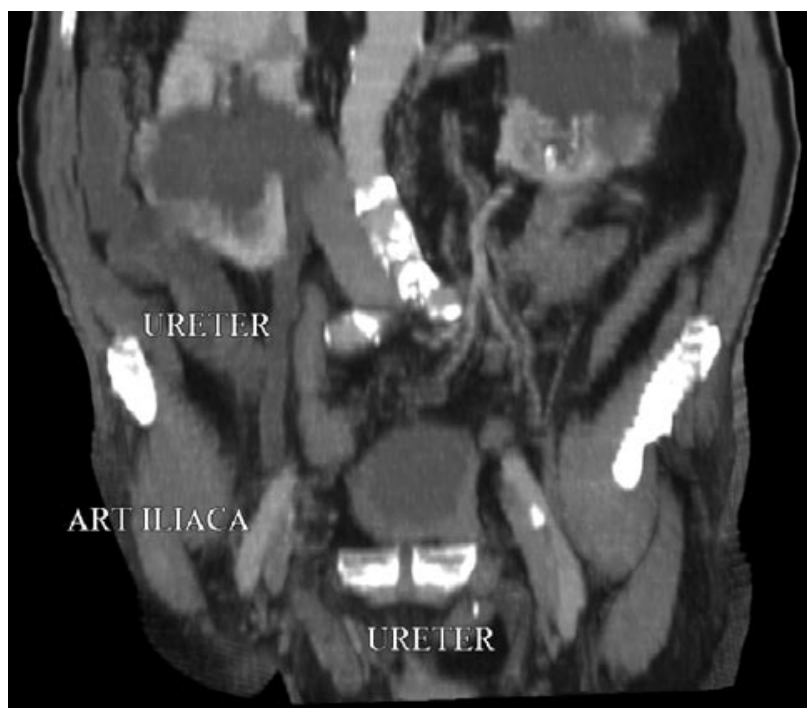

FIGURA 3. Reconstrucción coronal de TAC.

\section{CONCLUSIONES}

La herniación de uréter a través del canal inguinal es una entidad poco frecuente, generalmente diagnosticada de manera incidental. El tratamiento vendrá medido por la evaluación de los síntomas y posibles patologías asociadas al canal inguinal.

\section{BIBLIOGRAFIA y LECTURAS RECOMENDADAS (*lectura de interés $y^{* *}$ lectura fundamental)}

1. Pascale MD, Shabahang M, Evans SRT. Obstructive uropathy secodary to masive inguinioescrotal bladder herniation. J. Urol. 1993; 150:1906-8.

**2. Giglio M, Medica M, Germinale F, Raggio M, Campodonico F, Stubinski R, et al. Scrotal extraperitoneal hernia of the ureter: Case report and literatura review. Urol. Int. 2007; 66:166-8.

*3. Elider KS, Freedland SJ, Raifer J. Obstructive uropathy secondary to ureteroinguinal herniation. Rev. Urol. 2001; 3:207-8.

4. Tsai PJ, Lin JT, Wu TT, Tsai CC. Ureterosciatic hernia causes obstructive uropathy. J. Chin. Med. Assoc. 2008; 71:491-3.

5. Rommel FM, Boline GB, Huffnagle HW. Ureterosciatic hernia: an anatomical radigraphic correlation. J. Urol. 1993; 150:1232-3.

6. Pollac HM, Popky GL, Blumberg ML. Hernias of the uréter an anatomic-roentgenographic study. Radiology 1975; 117:275-81.

7. Marchal C, Chicharro JA, González JA, Ruiz JL, Burgos R. Unilateral ureteral hernia. Report of a case. Arch. Esp. Urol. 1994; 47(2):174-5. 